\title{
Appropriate Roles of Cardiac Troponins in Evaluating Patients With Chest Pain
}

\author{
Matthew S. Rice, MD, CPT, MC, USA, and David C. MacDonald, DO, MC, USA
}

Background: Diagnosis of acute myocardial infarction relies upon the clinical history, interpretation of the electrocardiogram, and measurement of serum levels of cardiac enzymes. Newer biochemical markers of myocardial injury, such as cardiac troponin $I$ and cardiac troponin $T$, are now being used instead of or along with the standard markers, the MB isoenzyme of creatine kinase (CK-MB) and lactate dehydrogenase.

Methods: We performed a MEDLINE literature search (1987 to 1997) using the key words "troponin I," "troponin T," and "acute myocardial infarction." We reviewed selected articles related to the diagnostic and prognostic usefulness of these cardiac markers in evaluating patients with suspected myocardial infarction.

Results: We found that (1) troponin I is a better cardiac marker than CK-MB for myocardial infarction because it is equally sensitive yet more specific for myocardial injury; (2) troponin $T$ is a relatively poorer cardiac marker than CK-MB because it is less sensitive and less specific for myocardial injury; and (3) both troponin $I$ and troponin $T$ may be used as independent prognosticators of future cardiac events.

Conclusions: Troponin I is a sensitive and specific marker for myocardial injury and can be used to predict the likelihood of future cardiac events. It is not much more expensive to measure than CK-MB. Overall, troponin I is a better cardiac marker than CK-MB and should become the preferred cardiac enzyme when evaluating patients with suspected myocardial infarction. (J Am Board Fam Pract 1999;12:214-8.)

The diagnosis of acute myocardial infarction depends upon the patient's clinical history, interpretation of the electrocardiogram (ECG), and measurement of serum levels of cardiac enzymes. Diagnostic uncertainty frequently arises because of a variety of factors. Many patients with acute myocardial infarction have atypical symptoms, and one half of patients with typical symptoms do not have acute myocardial infarction. One half of patients with acute myocardial infarction have nondiagnostic ECGs, and some patients are unable to provide a history. Biochemical markers of cardiac injury are therefore commonly relied upon to diagnose or exclude acute myocardial infarction., 1,2

An ideal serum marker would be both 100 percent sensitive to myocardial infarction (always detected in serum after acute myocardial infarction) and 100 percent specific to myocardial infarction

Submitted, revised, 16 July 1998.

From the Department of Family Practice (MSR, DCM), Madigan Army Medical Center, Fort Lewis, Wash. Address reprint requests to Matthew S. Rice, MD, 2109 Hammond Ave, DuPont, WA 98327.

The opinions or assertions contained herein are the private views of the authors and are not to be construed as official or reflecting the views of the Department of the Army or the Department of Defense. (detected in serum only after acute myocardial infarction). Such a marker would rise to detectable serum levels soon after acute myocardial infarction and would be simple and inexpensive to detect. Such an ideal marker would improve diagnostic clarity. Rapid and confident exclusion of acute myocardial infarction is important so that patients with noncardiac chest pain are not unnecessarily hospitalized for treatment (of course, other serious causes of chest pain should be excluded as well). Unfortunately, no such ideal marker is available.

Serum markers in current clinical use include the $\mathrm{MB}$ isoenzyme of creatine kinase (CK-MB), myoglobin, and cardiac troponins $T$ and $I$. These various proteins are released from myocardial cells after an insult, such as ischemia or infarct, and can be detected in the serum within hours of myocardial injury. Currently CK-MB is the serum cardiac marker relied upon as the diagnostic reference standard for acute myocardial infarction.

The diagnostic utility of CK-MB is limited, however, because it lacks specificity for myocardial injury (rendering false-positive results). Also, CKMB might fail to rise to abnormal levels in all patients with acute myocardial infarction, rendering 
Table 1. Sensitivities of Troponin T and CK-MB for Acute Myocardial Infarction, by Hours After Onset of Chest Pain.

\begin{tabular}{lcccccc}
\hline Marker & $3 \mathrm{~h}$ & $4 \mathrm{~h}$ & $5 \mathrm{~h}$ & $6 \mathrm{~h}$ & $7 \mathrm{~h}$ & $8 \mathrm{~h}$ \\
\hline Troponin T & 34 & 48 & 67 & 73 & 78 & 84 \\
CK-MB & 36 & 63 & 80 & 87 & 95 & 95 \\
\hline
\end{tabular}

From De Winter et al. ${ }^{4}$

false-negative results. The relatively brief duration of $\mathrm{CK}-\mathrm{MB}$ elevation following acute myocardial infarction limits its value as a cardiac marker in patients who have delayed symptoms. This article will examine two new serum markers-troponin $T$ and troponin I. The utility and clinical application of these markers will be discussed in regard to evaluating patients with chest pain.

\section{Physiologic Function of Cardiac Troponins}

The cardiac myofibril (the contractile element of the myocyte) is composed of two subunits, myosin and actin. The actin filament is composed of interwoven strands of F-actin and tropomyosin. Three loosely bound protein subunits (troponin I, troponin $T$, and troponin $C$ ) are attached to the tropomyosin filament. Troponin I has a strong affinity for actin; troponin $\mathrm{T}$ has a strong affinity for tropomyosin; and troponin $\mathrm{C}$ binds to calcium ions. In the presence of the troponin and tropomyosin complex, actin is inhibited from binding to myosin. When calcium ions are added, they bind to troponin C, and the troponin complex undergoes a conformational change, "tugging" on tropomyosin, thereby exposing the active sites on actin and allowing it to bind to myosin. All three troponins are integrally involved in the process of myofibril contraction and relaxation. ${ }^{3}$

\section{Cardiac Troponin T Sensitivity, Specificity, Serum Detection Time, and Prognostic Value Troponin T Is Not More Sensitive Than CK-MB \\ for Acute Myocardial Infarction}

De Winter et $\mathrm{al}^{4}$ studied the sensitivity of troponin $\mathrm{T}$ for acute myocardial infarction in 30 emergency department patients who had chest pain and found that the sensitivity of CK-MB was significantly better than that of troponin $\mathrm{T}$ in the early diagnosis of acute myocardial infarction (Table 1). Mair et $\mathrm{al}^{5}$ in a study of 37 patients found that within 4 hours of chest pain onset, the sensitivity of CK-MB (54 percent) was not signifi-
Table 2. Cumulative Sensitivities and Specificities of Troponin T and CK-MB for Retrospective Diagnosis of Acute Myocardial Infarction (12 to 48 Hours After Onset of Chest Pain).

\begin{tabular}{lcc}
\hline Marker & Sensitivity & Specificity \\
\hline Troponin T & 98.2 & 68.8 \\
CK-MB & 96.8 & 89.6 \\
\hline
\end{tabular}

From $W u$ and Lane. ${ }^{6}$

$\mathrm{CK}-\mathrm{MB}$ - $\mathrm{MB}$ isoenzyme of creatine kinase.

cantly different from that of troponin $T$ (46 percent).

In a meta-analysis of 8 troponin $\mathrm{T}$ studies, $\mathrm{Wu}$ and Lane ${ }^{6}$ found no significant difference between the sensitivities of troponin $T$ and CK-MB for retrospective diagnosis of acute myocardial infarction, 12 to 48 hours after onset of chest pain ('Table 2).

\section{Troponin T Is Not More Specific Tban CK-MB for Acute Myocardial Infarction}

Wu and Lane's meta-analysis ${ }^{6}$ found that CK-MB was significantly more specific than troponin $T$ for acute myocardial infarction (Table 2). De Winter et $\mathrm{al}^{4}$ in a study of 30 patients confirmed that the specificity of CK-MB (90 percent) was significantly better than that of troponin $T(80$ percent) at 24 hours after onset of chest pain.

The specificity of troponin $T$ for myocardial injury is limited because it is expressed in diseased and stressed skeletal muscle as well as in the cardiac myofibril. Although the troponin $\mathrm{T}$ gene is suppressed in healthy adult skeletal muscle, it is re-expressed in diseased (polymyositic, dystrophic) and chronically stressed skeletal muscle. Troponin $T$ is also found in the serum of uremic patients. Consequently, in patients with chest pain and concurrent chronic skeletal muscle injury, muscle disease, or kidney disease, troponin $T$ assays might yield falsepositive results. ${ }^{5,7}$

\section{Serum Levels of Troponin T Remain Elevated Longer Than Levels of CK-MB}

Both CK-MB and troponin $\mathrm{T}$ can be detected in serum within the first few hours after onset of acute myocardial infarction. CK-MB levels peak at 10 to 24 hours after acute myocardial infarction and disappear after 3 to 4 days. Troponin T levels peak at 14 hours after acute myocardial infarction, peak again several days later (biphasic peak), and 
remain abnormal for 10 days. ${ }^{1}$ This characteristic could make troponin $\mathrm{T}$ (in combination with CKMB) useful for retrospective diagnosis of acute myocardial infarction in patients who seek care very late.

\section{Troponin T Assays Are a Valuable Prognostic Tool}

Troponin $T$ is a good independent prognosticator of adverse outcomes in patients with unstable angina or non-Q-wave myocardial infarction. $\mathrm{Pa}$ tients with elevated troponin $T$ levels are at a much higher risk for future acute myocardial infarction, cardiac death, or the need for coronary revascularization; therefore, the troponin ' $T$ level might be useful as a patient risk-stratifying tool. ${ }^{8}$

\section{Cardiac Troponin I Sensitivity, Specificity, Serum Detection Time, and Prognostic Value \\ Troponin I Is at Least as Sersitive as CK-MB for Acute Myocardial Infarction}

Troponin I has been found to be a highly sensitive marker for acute myocardial infarction and is equal or superior to the sensitivity of CK-MB. This sensitivity improves with time after injury, reaching its plateau about 12 hours after acute myocardial infarction. Bodor et $\mathrm{al}^{9}$ and Cummins et $\mathrm{a}^{10,11}$ found that the sensitivity of CK-MB and troponin I were equivalent for acute myocardial infarction. The Bodor et al study of 30 patients who had acute myocardial infarction showed that the sensitivities of CK-MB and troponin I were both 93 percent by 12 hours after chest pain onset. In a study of 37 patients, Mair found that the sensitivity of troponin I was equivalent to that of CKMB for acute myocardial infarction within the first 4 hours after chest pain onset ( 49 and 54 percent, respectively). ${ }^{5}$ Cummins et al ${ }^{11}$ found abnormal levels of troponin I in all of 32 patients with acute myocardial infarction 12 hours after onset of chest pain, yielding a sensitivity of 100 percent. Adams et al, ${ }^{12}$ in a study of 89 patients with chest pain, found a troponin I sensitivity of 96.6 percent for acute myocardial infarction within 48 hours after chest pain onset.

In another study, Adams et al ${ }^{13}$ performed twodimensional echocardiography and measured serum levels of troponin I and CK-MB in $215 \mathrm{pa}-$ tients; including 37 with acute skeletal muscle trauma, 10 with chronic muscle disease (polymyositis, Duchenne muscular dystrophy, and idiopathic myopathy), 9 marathon runners, and 159 chronic dialysis patients. In this study, troponin I was elevated in every patient who was found to have regional wall abnormalities on echocardiogram (the reference standard for diagnosing acute myocardial infarction in patients with concurrent skeletal muscle trauma), yielding a 100 percent concordance with echocardiography for sensitivity to acute myocardial infarction.

\section{Troponin I Is Superior to CK-MB in Specificity for Acute Myocardial Infarction}

In the echocardiography study by Adams et al, ${ }^{13}$ CK-MB elevations were common among patients with skeletal muscle trauma and disease. Troponin I was not elevated in a single patient with skeletal muscle trauma, chronic muscle disease, or renal failure unless there was concurrent echocardiographic evidence of acute myocardial injury. Essentially, in this study, troponin I had a specificity of 100 percent for myocardial injury.

Whereas the specificity of troponin I appears to be superior to that of CK-MB, false-positives for acute myocardial infarction have been noted. It is believed, however, that these false-positives actually are markers of cardiac injury. Adams et $\mathrm{al}^{12}$ found elevated troponin I levels in only 5 of 99 chest pain patients without acute myocardial infarction, yielding a specificity of 95 percent. These 5 patients had unstable angina and possibly had subclinical infarcts. Bodor et $\mathrm{al}^{9}$ also found slightly elevated levels of troponin I in patients who had ischemic heart disease and proposed that small amounts of troponin I had leaked from ischemic myocardial cells or that those patients had small, clinically undetectable infarcts. Nevertheless, troponin I levels in the serum of ischemic patients were much lower than troponin I levels detected in the serum of patients who had an acute myocardial infarction. As with CK-MB, serum levels of troponin I directly correlate with the size and severity of the myocardial insult. ${ }^{11}$

The specificity of troponin I for myocardial injury is high because it has a unique amino acid sequence and is not expressed in either fetal skeletal muscle, acutely injured skeletal muscle, chronically diseased or stressed skeletal muscle, or in the serum of uremic patients. As a result, the troponin I assay can assist the clinician in diagnosing or excluding acute myocardial infarction in patients with concurrent uremia, skeletal muscle disease, or acute or chronic skeletal muscle trauma. ${ }^{14}$ 


\section{Levels of Troponin I Remain Elevated Longer Than Levels of $C K-M B$}

Several studies have shown that serum levels of both troponin I and CK-MB rise above normal within 5 to 7 hours after onset of acute myocardial infarction and reach peak serum levels at about 12 hours after acute myocardial infarction, but that troponin I remains elevated significantly longer than CK-MB. ${ }^{5,9-11}$ Like troponin T, troponin I has a second peak after several days. The first peak is due to the release of free troponin from the myocardial cytosol (where it is synthesized), and the second peak is from the release of bound troponin from degenerating myofibrils. Serum troponin I levels remain elevated for up to 8 days after acute myocardial infarction and could therefore replace lactate dehydrogenase (LDH) as a more specific long-term myocardial marker. ${ }^{11}$

\section{Troponin I Assays Are a Valuable Prognostic Tool}

As with troponin T, elevated troponin I levels predict a higher likelihood of future acute myocardial infarction or cardiac death; and troponin I can be used as a risk stratifier to select high-risk patients who are in need of more aggressive therapy, such as angioplasty or bypass grafting. ${ }^{15}$

\section{Summary}

\section{Troponin T}

For early diagnosis of acute myocardial infarction, troponin $\mathrm{T}$ has no diagnostic advantage to $\mathrm{CK}$ MB. The sensitivity of troponin $T$ is less than or equivalent to that of $\mathrm{CK}-\mathrm{MB}$; the specificity of troponin $T$ is impaired because troponin $T$ is expressed in the serum of patients with uremia or damaged or diseased skeletal muscle; and the time to serum detection for troponin $T$ is equivalent to that for CK-MB. Troponin $\mathrm{T}$ can be used as a prognostic tool and risk stratifier. Although the laboratory turnaround time for both tests is about 2 hours, the cost for a troponin $T$ assay is substantially more than the cost of a CK-MB assay ( $\$ 41$ versus \$9) (Madigan Army Medical Center chemistry laboratory).

\section{Troponin I}

Current studies suggest that compared with CK$\mathrm{MB}$, troponin I offers considerable advantages in the early diagnosis of acute myocardial infarction. The sensitivity of troponin I is equivalent to that of $\mathrm{CK}-\mathrm{MB}$, and troponin I is much more specific for myocardial injury. An abnormal troponin I level is therefore more likely to represent true infarction. Troponin I serum detection time is equivalent to that of CK-MB. Troponin I is a prognostic indicator of future infarction or cardiac death. The cost of a troponin I assay (\$15) is slightly more than the cost of a CK-MB assay (\$9), and the laboratory turnaround time for both tests is about 2 hours (Madigan Army Medical Center chemistry laboratory). Troponin I levels determined in the emergency department add higher specificity for myocardial injury without sacrificing sensitivity, time, or an undue amount of money.

\section{Addendum}

At this time, newer point-of-care serum assays for $\mathrm{CK}-\mathrm{MB}$, troponins, and myoglobin are available. These enable rapid (within minutes) serum testing for cardiac markers.

\section{References}

1. Rozenman Y, Gotsman MS. The earliest diagnosis of acute myocardial infarction. Annu Rev Med 1994; 45:31-44.

2. Newby LK, Gibler WB, Ohman EM, Christenson RH. Biochemical markers in suspected acute myocardial infarction: the need for early assessment. Clin Chem 1995;41:1263-5.

3. Guyton AC. Textbook of medical physiology, 8th ed. Philadelphia: WB Saunders, 1991:71-2.

4. de Winter RJ, Koster RW, Sturk A, Sanders GT. Value of myoglobin, troponin $T$, and CK-MB mass in ruling out an acute myocardial infarction in the emergency room. Circulation 1995;92:3401-7.

5. Mair J, Morandell D, Genser N, Lechleimer P, Dienstl F, Puschendorf B. Equivalent early sensitivities of myoglobin, creatine kinase $\mathrm{MB}$ mass, creatine kinase isoform ratios, and cardiac troponins $I$ and $T$ for acute myocardial infarction. Clin Chem 1995;41: 1266-72.

6. Wu AH, Lane PL. Metaanalysis in clinical chemistry: validation of cardiac troponin $T$ as a marker for ischemic heart diseases. Clin Chem 1995;41(8 Pt 2):1228-33.

7. Keffer JH. Myocardial markers of injury. Evolution and insights. Am J Clin Pathol 1996;105:305-20.

8. Ohman EM, Armstrong PW, Christenson RH, Granger CB, Katus HA, Hamm CW, et al. Cardiac troponin $\mathrm{T}$ levels for risk stratification in acute myocardial ischemia. GUSTO IIA Investigators. N Engl J Med 1996;335:1333-41.

9. Bodor GS, Porter S, Landt Y, Ladenson JH. The development of monoclonal antibodies and an assay for cardiac troponin-I with preliminary results in 
suspected cases of myocardial infarction. Clin Chem 1992;38:2203-14.

10. Cummins B, Cummins P. Cardiac specific troponin-I release in canine experimental myocardial infarction: development of a sensitive enzyme-linked immunoassay. J Mol Cell Cardiol 1987;19:999-1010.

11. Cummins B, Auckland ML, Cummins P. Cardiacspecific troponin-I radioimmunoassay in the diagnosis of acute myocardial infarction. Am Heart J 1987; 113:1333-44.

12. Adams JE 3rd, Schechtman KB, Landt Y, Ladenson $\mathrm{JH}$, Jaffe AS. Comparable detection of acute myocardial infarction by creatine kinase MB isoenzyme and cardiac troponin I. Clin Chem 1994;40(7 Pt 1):1291-5.

13. Adams JE 3rd, Bodor GS, Davila-Roman VG, Delmez JA, Apple FS, Ladenson JH, et al. Cardiac troponin I. A marker with high specificity for cardiac injury. Circulation 1993;88:101-6.

14. Jaffe AS. In search of specificity: the troponins. Acc Curr J Rev 1995;5:29-33.

15. Antman EM, Tanasijevic MJ, Thompson B, Schactman M, McCabe CH, Cannon CP, et al. Cardiacspecific troponin I levels to predict the risk of mortality in patients with acute coronary syndromes. $\mathrm{N}$ Engl J Med 1996;335:1342-9. 